Calculus of variations manuscript No.

(will be inserted by the editor)

Filippo Gazzola $\cdot$ Hans-Christoph Grunau · Marco Squassina

\title{
Existence and nonexistence results for critical growth biharmonic elliptic equations
}

Received: date / Accepted: date /

Published online: date - (c) Springer-Verlag 2002

\begin{abstract}
We prove existence of nontrivial solutions to semilinear fourth order problems at critical growth in some contractible domains which are perturbations of small capacity of domains having nontrivial topology. Compared with the second order case, some difficulties arise which are overcome by a decomposition method with respect to pairs of dual cones. In the case of Navier boundary conditions, further technical problems have to be solved by means of a careful application of concentration compactness lemmas. The required generalization of a Struwe type compactness lemma needs a somehow involved discussion of certain limit procedures.

Also nonexistence results for positive solutions in the ball are obtained, extending a result of Pucci and Serrin on so-called critical dimensions to Navier boundary conditions. A Sobolev inequality with optimal constant and remainder term is proved, which is closely related to the critical dimension phenomenon. Here, this inequality serves as a tool in the proof of the existence results and in particular in the discussion of certain relevant energy levels.
\end{abstract}

Mathematics Subject Classification (2000): 35J65; 35J40, 58E05

\section{Introduction}

We consider the following biharmonic critical growth problem

$$
\Delta^{2} u=\lambda u+|u|^{2_{*}-2} u \quad \text { in } \Omega
$$

either with Navier boundary conditions

$$
u=\Delta u=0 \quad \text { on } \partial \Omega
$$

F. Gazzola: Dipartimento di Scienze e Tecnologie Avanzate, Corso Borsalino 54, I-15100 Alessandria, Italy. e-mail: gazzola@unipmn.it

H.-Ch. Grunau: Fakultät für Mathematik der Universität, Postfach 4120,

D-39016 Magdeburg, Germany. e-mail: grunau@mathematik.uni-magdeburg.de

M. Squassina: Dipartimento di Matematica e Fisica

Via Musei 41, I-25121 Brescia, Italy. e-mail: squassin@dmf.bs.unicatt.it

The first author was supported by MURST project "Metodi Variazionali ed Equazioni Differenziali non Lineari" 
or with Dirichlet boundary conditions

$$
u=\nabla u=0 \quad \text { on } \partial \Omega .
$$

Here $\Omega \subset \mathbb{R}^{n}(n \geq 5)$ is a bounded smooth domain, $\lambda \geq 0$ and $2_{*}=\frac{2 n}{n-4}$ is the critical Sobolev exponent for the embedding $H^{2}\left(\mathbb{R}^{n}\right) \hookrightarrow L^{2 *}\left(\mathbb{R}^{n}\right)$. We are interested in existence and nonexistence of nontrivial solutions of (1) with one of the above boundary conditions.

We consider first the case where $\lambda=0$, namely the equation

$$
\Delta^{2} u=|u|^{8 /(n-4)} u \quad \text { in } \Omega .
$$

It is well known that (4)-(2) and (4)-(3) admit no positive solutions if $\Omega$ is star shaped (see [27, Theorem 3.3] and [30, Corollary 1]). Clearly, these are not exhaustive nonexistence results as they only deal with positive solutions, see also Section 3 below for further comments. On the other hand, by combining the Pohožaev identity [34] with the unique continuation property [22, Section 3], it is known that one indeed has nonexistence results of any nontrivial solution for the correponding second order equation $-\Delta u=|u|^{4 /(n-2)} u$ in star shaped domains. This suggests that, in order to obtain existence results for (4), one should either add subcritical perturbations like the linear term $\lambda u$ in (1) or modify the topology or the geometry of the domain $\Omega$. For general subcritical perturbations we refer to $[4,13]$ and references therein. Domains with nontrivial topology are studied in [3] for Dirichlet and in [11] for Navier boundary conditions. Here we are interested in topologically simple but geometrically complicated domains. As far as we are aware, the case of "strange" contractible domains has not been tackled before and this is precisely the first aim of the present paper. We show that (4)-(2) admits a positive solution in a suitable contractible domain $\Omega$. This result is the exact counterpart of [27, Theorem 3.3] where it is shown that (4)-(2) admits no positive solution if $\Omega$ is star shaped. For the Dirichlet boundary condition we obtain a weaker result: we show that (4)-(3) admits a nontrivial solution in the same kind of contractible domain. Clearly, this still leaves open some problems concerning Dirichlet boundary conditions, see Section 3. On one hand our proof is inspired by strong arguments developed by Passaseo [32], on the other hand we have to face several hard difficulties, especially (and somehow unexpectedly) under Navier boundary conditions. One of the crucial steps in the approach by Passaseo is to prove that sign changing solutions of (4) "double the energy" of the associated functional. For the second order problem this may be shown by the usual technique of testing the equation with the positive and negative parts of the solution. Of course, this technique fails for (4) where higher order derivatives are involved and we overcome this difficulty thanks to the decomposition method in dual cones developed in [14]. This method enables us to bypass the lack of nonexistence results for nodal solutions of (4) in star shaped domains. Moreover, when dealing with Navier boundary conditions, the required generalization of the Struwe compactness lemma [37] turns out to 
be very delicate because of the second boundary datum and the lack of a uniform extension operator for $H^{2} \cap H_{0}^{1}$-functions in families of domains. See Lemma 5 and its proof in Section 7. The same problem arises in Lemma 7 where a uniform lower bound for an enlarged optimal Sobolev constant has to be found in a suitable class of domains. An important tool for this Lemma is a Sobolev inequality with optimal constant and remainder term, see Theorem 5. This inequality is closely related to nonexistence results, which will be summarized in what follows.

Next, we restrict our attention to the case where $\lambda>0$ and $\Omega=B$, the unit ball in $\mathbb{R}^{n}$. We are interested in positive radially symmetric solutions. Let $\lambda_{1}>0$ be the first eigenvalue of $\Delta^{2}$ with homogeneous Dirichlet boundary conditions. A celebrated result by Pucci-Serrin [36] shows that (1)-(3) admits a nontrivial radially symmetric solution for all $\lambda \in\left(0, \lambda_{1}\right)$ if and only if $n \geq 8$ ( $n$ is the space dimension). If $n=5,6,7$ the range $0<\lambda<\lambda_{1}$ is no longer correct since there exists $\lambda_{*} \in\left(0, \lambda_{1}\right)$ such that (1)-(3) admits no nontrivial radial solution whenever $\lambda \in\left(0, \lambda_{*}\right]$; moreover, there exists $\lambda^{*} \in\left[\lambda_{*}, \lambda_{1}\right)$ (presumably $\lambda^{*}=\lambda_{*}$ ) such that (1)-(3) admits even a positive radially symmetric solution for all $\lambda \in\left(\lambda^{*}, \lambda_{1}\right)$. In other words, the well known [6] nonexistence result for radially symmetric solutions of the second order problem for small $\lambda$ in dimension $n=3$ carries over to (1)-(3) in dimensions $n=5,6,7$ : Pucci-Serrin call these dimensions critical. Furthermore, the space dimensions $n$ for which one has nonexistence of positive radially symmetric solutions of (1)-(3) for $\lambda$ in some (right) neighbourhood of 0 are called weakly critical [17]. In Theorem 3 below we prove that the dimensions $n=5,6,7$ are weakly critical also for Navier boundary conditions. Therefore, critical dimensions seem not to depend on the boundary conditions. This result is perhaps related to the fact that critical dimensions may have an explanation in terms of the summability of the fundamental solution corresponding to the differential operator $\Delta^{2}$, see $[20,26]$.

Finally, when $\Omega=B$ we show that (4)-(3) admits no nontrivial radially symmetric solution; note that no positivity assumptions on the solution are made. This result was already obtained in [16, Theorem 3.11]; we give here a different (and simpler) proof.

\section{Existence results}

By $D^{2,2}\left(\mathbb{R}^{n}\right)$ we denote the completion of the space $C_{c}^{\infty}\left(\mathbb{R}^{n}\right)$ with respect to the norm $\|f\|_{2,2}^{2}=\int_{\mathbb{R}^{n}}|\Delta f|^{2}$; this is a Hilbert space when endowed with the scalar product

$$
(f, g)_{2,2}=\int_{\mathbb{R}^{n}} \Delta f \Delta g d x .
$$

By solution of (1) we mean here a function $u \in H^{2} \cap H_{0}^{1}(\Omega)$ (when dealing with (2)) or a function $u \in H_{0}^{2}(\Omega)$ (when dealing with (3)) which satisfies

$$
\int_{\Omega} \Delta u \Delta \varphi d x=\int_{\Omega}\left(\lambda u \varphi+|u|^{8 /(n-4)} u \varphi\right) d x
$$


for all $\varphi \in H^{2} \cap H_{0}^{1}(\Omega)$ (resp. $H_{0}^{2}(\Omega)$ ). For Dirichlet boundary conditions this variational formulation is standard, while for Navier boundary data we refer to [4, p.221]. By well known regularity results (see [25, Theorem 1] and [43, Lemma B3]), if $\partial \Omega \in C^{4, \alpha}$, then the solutions $u$ of (1) satisfy $u \in C^{4, \alpha}(\bar{\Omega})$ and solve $(1)$ in the classical sense.

Definition 1. Let $K \subset \mathbb{R}^{n}$ bounded. We say that $u \in D^{2,2}\left(\mathbb{R}^{n}\right)$ satisfies $u \geq 1$ on $K$ in the sense of $D^{2,2}\left(\mathbb{R}^{n}\right)$ if there exists a sequence $\left(u_{h}\right)$ in $C_{c}^{2}\left(\mathbb{R}^{n}\right)$ such that $u_{h} \geq 1$ on $K$ for each $h \in \mathbb{N}$ and $u_{h} \rightarrow u$ in $D^{2,2}\left(\mathbb{R}^{n}\right)$. Analogously, $u \leq 1$ and $u=1$ on $K$ are defined.

Definition 2. We define the $(2,2)$-capacity of $K$ (cap $K$ ) as

$$
\operatorname{cap} K=\inf \left\{\int_{\mathbb{R}^{n}}|\Delta u|^{2} d x: u=1 \text { on } K \text { in the } D^{2,2}\left(\mathbb{R}^{n}\right) \text { sense }\right\} \text {. }
$$

We set $\operatorname{cap} \emptyset:=0$.

Since the nonempty set

$$
\left\{u \in D^{2,2}\left(\mathbb{R}^{n}\right): u=1 \text { on } K \text { in the } D^{2,2}\left(\mathbb{R}^{n}\right) \text { sense }\right\}
$$

is closed and convex, there exists a unique function $z_{K} \in D^{2,2}\left(\mathbb{R}^{n}\right)$ such that $z_{K}=1$ on $K$ and

$$
\int_{\mathbb{R}^{n}}\left|\Delta z_{K}\right|^{2} d x=\operatorname{cap} K
$$

Finally we make precise what we mean by set deformations:

Definition 3. Let $\widetilde{\Omega} \subset \mathbb{R}^{n}$ and let $H, \Omega \subset \widetilde{\Omega}$. We say that $H$ can be deformed in $\widetilde{\Omega}$ into a subset of $\Omega$ if there exists a continuous function

$$
\mathscr{H}: H \times[0,1] \rightarrow \widetilde{\Omega}
$$

such that $\mathscr{H}(x, 0)=x$ and $\mathscr{H}(x, 1) \in \Omega$ for all $x \in H$.

Our first result states the existence of positive solutions for the critical growth equation (4) with Navier boundary conditions. Combined with the already mentioned nonexistence result in star shaped domains [27], this shows that the existence of positive solution strongly depends on the geometry of the domain.

Theorem 1. Let $\widetilde{\Omega}$ be a smooth bounded domain of $\mathbb{R}^{n}(n \geq 5)$ and let $H$ be a closed subset contained in $\widetilde{\Omega}$. Then there exists $\varepsilon>0$ such that if $\Omega \subset \widetilde{\Omega}$ is a smooth domain with $\operatorname{cap}(\widetilde{\Omega} \backslash \Omega)<\varepsilon$ and such that $H$ cannot be deformed in $\widetilde{\Omega}$ into a subset of $\Omega$ then there exists a positive solution of

$$
\begin{cases}\Delta^{2} u=u^{(n+4) /(n-4)} & \text { in } \Omega \\ u=\Delta u=0 & \text { on } \partial \Omega .\end{cases}
$$


We will prove this result in Section 5 .

Next we turn to Dirichlet boundary conditions. In this case, we merely show the existence of nontrivial solutions to (4). The lack of information about the sign of the solution is due to the lack of information about the sign of the corresponding Green's function. Moreover, in general domains sign change has even to be expected. See [18] for a survey on this feature and for further references.

Theorem 2. Let $\widetilde{\Omega}$ be a smooth bounded domain of $\mathbb{R}^{n}(n \geq 5)$ and let $H$ be a closed subset contained in $\widetilde{\Omega}$. Then there exists $\varepsilon>0$ such that if $\Omega \subset \widetilde{\Omega}$ is a smooth domain with $\operatorname{cap}(\widetilde{\Omega} \backslash \Omega)<\varepsilon$ and such that $H$ cannot be deformed in $\widetilde{\Omega}$ into a subset of $\Omega$ then there exists a nontrivial solution of

$$
\begin{cases}\Delta^{2} u=|u|^{8 /(n-4)} u & \text { in } \Omega \\ u=\nabla u=0 & \text { on } \partial \Omega .\end{cases}
$$

The proof of this result is simpler than the one of Theorem 1. As we will explain below, for (5), one has to study very carefully the behaviour of suitable sequences $u_{h} \in H^{2} \cap H_{0}^{1}\left(\Omega_{h}\right)$ for varying domains $\Omega_{h}$. In contrast with the spaces $H_{0}^{2}\left(\Omega_{h}\right)$, there is no "trivial" extension operator into $H^{2}\left(\mathbb{R}^{n}\right)$. Only as positivity of solutions is concerned, the situation with respect to Dirichlet boundary conditions is more involved than with respect to Navier boundary conditions. Here we have no positivity statement of the solution because Lemma 3 below does not seem to hold.

If $\Omega$ is an annulus and if one restricts to radial functions, the problem is no longer critical and then both (5) and (6) admit a nontrivial solution. For the second order problem $-\Delta u=|u|^{4 /(n-2)} u$ in $\Omega, u=0$ on $\partial \Omega$, it was shown with help of very subtle methods by Coron [7] and Bahri-Coron [2] that this result extends to domains with nontrivial topology. Recently, corresponding results have been found also in the higher order case: see [11] for problem (5) and [3] for the polyharmonic analogue of (6) under Dirichlet boundary conditions. However, the solutions which are constructed in these papers and here are not related. To explain this, assume that $\widetilde{\Omega}$ satisfies the assumptions of [11] or [3]. If $u_{\Omega}$ is the solution of (5) or (6), then by exploiting the proof of Theorem 1 one has that $u_{\Omega}$ converges weakly to zero as $\operatorname{cap}(\widetilde{\Omega} \backslash \Omega) \rightarrow 0$. Hence, any nontrivial solution in $\widetilde{\Omega}$ may not be obtained as limit of the solutions $u_{\Omega}$ in $\Omega$. On the other hand one expects the nontrivial solutions in $\widetilde{\Omega}$ to be stable and to remain under "small" perturbations $\Omega \subset \widetilde{\Omega}$. These solutions in $\Omega$ will be different from ours. The latter situation was studied in [9] for the second order problem.

To conclude the section, we observe that there are also contractible domains $\Omega$, which satisfy the assumptions of the preceding theorems. In the following example we describe precisely such a situation. Further examples may be adapted to the biharmonic setting from [32, pp. 39-41].

Example 1. We consider an annular shaped domain as $\widetilde{\Omega} \subset \mathbb{R}^{n}$ with $n>5$, where we drill a sufficiently "thin" cylindrical hole along a straight line in order to obtain the smooth contractible subdomain $\Omega$. 
To be more precise: we assume that for $\varepsilon$ small enough, $\widetilde{\Omega} \backslash \Omega_{\varepsilon}$ is contained in a cylinder with basis $B_{\varepsilon} \subset \mathbb{R}^{n-1}$ and fixed height. Then by simple scaling arguments one finds that $\operatorname{cap}\left(\widetilde{\Omega} \backslash \Omega_{\varepsilon}\right)=O\left(\varepsilon^{n-5}\right) \rightarrow 0$ as $\varepsilon \rightarrow 0$, provided that the dimension satisfies $n>5$.

We choose $H$ to be a spherical hypersurface in $\widetilde{\Omega}$, which cannot be deformed into a subset of $\Omega_{\varepsilon}$. This can be seen by looking at the degree of mapping $d(\mathscr{H}(., t), H, 0)$ for $t \in[0,1]$, where $\mathscr{H}$ is assumed to exist according to Definition 3.

Instead of the above mentioned segment of fixed length, one may consider any bounded piece of a fixed generalized plane, provided that its codimension is at least 5 .

\section{Nonexistence results and a Sobolev inequality with optimal constant and remainder term}

We collect here a number of known as well as of new nonexistence results for the equation

$$
\Delta^{2} u=\lambda u+|u|^{8 /(n-4)} u \text { in } \Omega
$$

under either Navier or Dirichlet boundary conditions. Here, we include the linear term $\lambda u$. It turns out that the discussion of the borderline case $\lambda=0$, where the purely critical equation has to be considered, is somehow involved and still not exhaustive. This lack of nonexistence results complicates the formulation of the compactness result Lemma 5 below.

The functional analytic counterpart of nonexistence results for (7) for small $\lambda>0$ in so-called critical dimensions are Sobolev inequalities with optimal constant and remainder term. (This becomes clear in the proof of Theorem 5. See also [14].) Such an inequality in $H_{0}^{2}(\Omega)$ - i.e. under Dirichlet boundary conditions - was proved in [14] while the result in $H^{2} \cap H_{0}^{1}(\Omega)$ i.e. under Navier boundary conditions - is provided in Sect. 3.3 below. In the present paper, this refined Sobolev inequality is applied to show that the optimal Sobolev constant can be increased uniformly for all subdomains $\Omega \subset \widetilde{\Omega}$ with help of a condition on the "barycenter" of the functions $u \in$ $H^{2} \cap H_{0}^{1}(\Omega)$. See Lemma 7 below.

\subsection{Nonexistence under Navier boundary conditions}

It turns out that nonexistence questions in this case are relatively hard and that only restricted results are available.

The case $\lambda=0$

In this case, no positive solution to (5) may exist, provided $\Omega$ is star shaped. See [42, Theorem 3.10] and also [27, Theorem 3.3]. The key ingredient is a Pohožaev type identity [35]. By means of techniques developed by Pucci and Serrin in [36], such a nonexistence result may be also shown for nontrivial radial solutions in the ball. 
More difficult seems this nonexistence result for any nontrivial solution of (5). In this case the Pohožaev type identity is not powerful enough to extend $u$ outside $\Omega$ by zero as a solution of (4) in $\mathbb{R}^{n}$ and then to apply the unique continuation property [33].

The case $\lambda>0$

In Section 6 we prove the following result.

Theorem 3. Let $n \in\{5,6,7\}$ and $B \subset \mathbb{R}^{n}$ be the unit ball. Then there exists a number $\lambda_{*}>0$ such that the problem

$$
\begin{cases}\Delta^{2} u=\lambda u+u^{(n+4) /(n-4)} & \text { in } B \\ u=\Delta u=0 & \text { on } \partial B\end{cases}
$$

admits no positive solution if $\lambda \in\left(0, \lambda_{*}\right]$.

By [41], positive solutions to (8) are radially symmetric.

Theorem 3 answers a question left open by van der Vorst in [44, Theorem 3]. Moreover, in [44, Theorem 1] complementary existence results were shown implying that such a nonexistence result can hold at most in the dimensions $5,6,7$.

These are the critical dimensions for problem (8), at least, when restricting to positive solutions in the ball. Theorem 3 can also be shown just for nontrivial radial solutions: here one has to combine methods of [36] with some techniques, which appear in the proof of Theorem 4 below.

Again, nonexistence of any nontrivial solution, although expected, has to be left open.

The case $\lambda<0$

In the preceding nonexistence results, the positivity of a solution, the nonexistence of which had to be shown, is intensively exploited. For $\lambda \geq 0$ one can conclude from $u \geq 0$ that even $-\Delta u \geq 0$ in $\Omega$.

For $\lambda$ negative, we can only argue as before, when $\lambda$ is close enough to 0 , see $[28,21]$. If $\lambda<<0$, the framework of positive solutions is no longer adequate for (7), and the argument in [42, pp. 390/391] and [44, Theorem 3] breaks down. Instead, one has to look for nonexistence of any nontrivial solution. But such a result seems to be still unknown, even for nontrivial radial solutions in the ball.

\subsection{Nonexistence under Dirichlet boundary conditions}

In this situation, existence results for positive solutions are more involved than under Navier boundary conditions (see [15]) and cannot even be expected in general domains. On the other hand, we know much more about nonexistence for relatively large classes of solutions.

The case $\lambda<0$

Nonexistence of any nontrivial solution is shown in [35], provided the domain $\Omega$ is star shaped. This proof is based on a generalized Pohožaev identity 
and covers also the general polyharmonic problem with $(-\Delta)^{K}$ as linear principal part.

The case $\lambda>0$

In [36] we already find the following result, which gives a stronger statement than the analogous Theorem 3 above for Navier boundary conditions:

Let $n \in\{5,6,7\}$ and assume that $\Omega$ is the unit ball $B$. Then there exists a number $\lambda_{*}>0$ such that a necessary condition for a nontrivial radial solution to (6) to exist is $\lambda>\lambda_{*}$.

As this special behaviour of the critical growth Dirichlet problem (6) may be observed only in space dimensions 5, 6 and 7, Pucci and Serrin call these dimensions critical. For the critical dimension phenomenon for general polyharmonic problems with Dirichlet boundary conditions we refer to [17].

The case $\lambda=0$

Also in this case, the nonexistence of any nontrivial solution to (6) has to be left open, only more restricted results are available. Oswald [30] proved the nonexistence of positive solutions in strictly star shaped domains by means of a Pohožaev type identity (see e.g. [27, (2.6)]) and strong maximum principles for superharmonic functions.

Moreover, we can also exclude nontrivial radial solutions:

Theorem 4. Let $B \subset \mathbb{R}^{n}(n \geq 5)$ be the unit ball. Then problem

$$
\begin{cases}\Delta^{2} u=|u|^{8 /(n-4)} u & \text { in } B, \\ u=\nabla u=0 & \text { on } \partial B\end{cases}
$$

admits no nontrivial radial solution.

This result can be found in [16, Theorem 3.11]. The proof there is based on a refined integral identity of Pucci and Serrin [36] and some Hardy type embedding inequalities. In Section 6 we will give a more elementary and geometric proof.

Unfortunately neither this proof nor the one given in [16, Theorem 3.11] carry over to the general polyharmonic problem, where the discussion of nonexistence of nontrivial radial solutions in the borderline case $\lambda=0$ remains essentially open.

\subsection{An optimal Sobolev inequality with remainder term}

Sobolev embeddings with critical exponents and the corresponding optimal Sobolev constants will play a crucial role in the proof of our existence results.

Let us set

$$
S=\inf _{u \in D^{2,2}\left(\mathbb{R}^{n}\right) \backslash\{0\}} \frac{\|\Delta u\|_{2}^{2}}{\|u\|_{2_{*}}^{2}} .
$$


When working in the whole $\mathbb{R}^{n}$, this optimal Sobolev constant is attained. According to Lemma 2 below, minimizers are necessarily of one sign. Positive solutions of the corresponding Euler Lagrange equations are known to be of the following form.

Lemma 1. The constant $S$ in (10) is a minimum and (up to nontrivial real multiples) it is attained only by the functions

$$
u_{\varepsilon, x_{0}}(x)=\frac{[(n-4)(n-2) n(n+2)]^{(n-4) / 8} \varepsilon^{(n-4) / 2}}{\left(\varepsilon^{2}+\left|x-x_{0}\right|^{2}\right)^{(n-4) / 2}},
$$

for any $x_{0} \in \mathbb{R}^{n}$ and each $\varepsilon>0$. Moreover, the functions $u_{\varepsilon, x_{0}}$ are the only positive solutions of the equation

$$
\Delta^{2} u=u^{(n+4) /(n-4)} \quad \text { in } \mathbb{R}^{n} .
$$

Proof. From Lemma 2 below, we take that any minimizer of (10) may be assumed to be positive. Then, the result follows from [12, Theorem 2.1], [39, Theorem 4] and [23, Theorem 1.3].

Similarly one can define $S(\Omega)$ for any $\Omega \subset \mathbb{R}^{n}$. It is well known that $S(\Omega)$ is not attained if $\Omega$ is bounded [12] and that it does not depend neither on the domain nor on the boundary conditions [43]. The following extension of the embedding inequality corresponding to (10) with optimal constant $S$ gives a quantitative formulation for the fact that $S$ is not attained. Here, it will be used to show Lemma 7, which in turn is an important step in the proof of our existence results.

Theorem 5. Let $\Omega \subset \mathbb{R}^{n}$ be open and of finite measure and $p \in\left[1, \frac{n}{n-4}\right)$. Then there exists a constant $C=C(n, p,|\Omega|)>0$, such that for every $u \in H^{2} \cap H_{0}^{1}(\Omega)$, one has

$$
\int_{\Omega}|\Delta u|^{2} d x \geq S\|u\|_{2_{*}}^{2}+\frac{1}{C}\|u\|_{p}^{2}
$$

Here, $|\Omega|$ denotes the $n$-dimensional Lebesgue measure of $\Omega$. Moreover, if $\omega>0$, then (13) holds with a constant $C=C(n, p, \omega)$ for all $\Omega$ with $|\Omega| \leq \omega$.

For the smaller class $H_{0}^{2}(\Omega)$ a more refined result can be found in [14]. The proof there is based on an extension and decomposition method (see also Section 4 below), by means of which analogues to (13) for the spaces $H_{0}^{m}(\Omega)$ of any order can be also obtained. This proof, however, does not seem to carry over directly to the space $H^{2} \cap H_{0}^{1}$ due to the lack of information at the boundary. In Section 6 we sketch a technical proof of Theorem 5 , which is based on nonexistence results like in Theorem 3 and on Talenti's symmetrization result [40]. 


\section{Preliminary results}

In the previous section we have briefly discussed energy minimizing solutions of (12). These are necessarily of one sign (this is obtained as a byproduct of the following lemma) and of the form given in Lemma 1. The next results show that nodal solutions of (4) double the energy level of the corresponding "free" energy functional (observe that here, we are working with a constrained functional). This will allow us to bypass the lack of nonexistence results for nodal solutions of (4) in star shaped domains.

Lemma 2. Let $u \in D^{2,2}\left(\mathbb{R}^{n}\right)$ be a nodal solution of the equation

$$
\Delta^{2} u=|u|^{8 /(n-4)} u \quad \text { in } \mathbb{R}^{n} \text {. }
$$

Then $\|\Delta u\|_{2}^{2} \geq 2^{4 / n} S\|u\|_{2_{*}}^{2}$.

Proof. Consider the convex closed cone

$$
\mathscr{K}=\left\{u \in D^{2,2}\left(\mathbb{R}^{n}\right): u \geq 0 \text { a.e. in } \mathbb{R}^{n}\right\},
$$

and its dual cone

$$
\mathscr{K}^{\prime}=\left\{u \in D^{2,2}\left(\mathbb{R}^{n}\right): \quad(u, v)_{2,2} \leq 0 \quad \forall v \in \mathscr{K}\right\} .
$$

Let us show that $\mathscr{K}^{\prime} \subseteq-\mathscr{K}$. For each $h \in C_{c}^{\infty}\left(\mathbb{R}^{n}\right) \cap \mathscr{K}$ consider the solution $u_{h}$ of the problem

$$
\Delta^{2} u_{h}=h \quad \text { in } \mathbb{R}^{n}
$$

Then by the positivity of the fundamental solution of $\Delta^{2}$ in $\mathbb{R}^{n}$, it follows $u_{h} \in \mathscr{K}$ and thus if $v \in \mathscr{K}^{\prime}$,

$$
\forall h \in C_{c}^{\infty}\left(\mathbb{R}^{n}\right) \cap \mathscr{K}: \int_{\mathbb{R}^{n}} h v d x=\int_{\mathbb{R}^{n}} \Delta^{2} u_{h} v d x=\left(u_{h}, v\right)_{2,2} \leq 0 .
$$

By a density argument one obtains $v \leq 0$ a.e. in $\mathbb{R}^{n}$, i.e. $v \in-\mathscr{K}$. Now, by a result of Moreau [29], for each $u \in D^{2,2}\left(\mathbb{R}^{n}\right)$ there exists a unique pair $\left(u_{1}, u_{2}\right)$ in $\mathscr{K} \times \mathscr{K}^{\prime}$ such that

$$
u=u_{1}+u_{2}, \quad\left(u_{1}, u_{2}\right)_{2,2}=0 .
$$

Let $u$ be a nodal solution of (14) and let $u_{1} \in \mathscr{K}$ and $u_{2} \in \mathscr{K}^{\prime}$ be the components of $u$ according to this decomposition. We obtain that $u_{i} \not \equiv 0$ and

$$
|u(x)|^{2_{*}-2} u(x) u_{i}(x) \leq\left|u_{i}(x)\right|^{2_{*}}, \quad i=1,2,
$$

for a.e. $x \in \mathbb{R}^{n}$. Indeed, if $i=1$ and $u(x) \leq 0$ then (16) is trivial, while if $u(x) \geq 0$, since $u_{2} \in-\mathscr{K}$ one has $u(x)=u_{1}(x)+u_{2}(x) \leq u_{1}(x)$ and again 
(16) holds. The case $i=2$ is similar. By combining the Sobolev inequality with (15) and (16), we get for $i=1,2$

$$
\begin{aligned}
& S\left\|u_{i}\right\|_{2_{*}}^{2} \leq\left\|u_{i}\right\|_{2,2}^{2}=\int_{\mathbb{R}^{n}} \Delta u \Delta u_{i} d x=\int_{\mathbb{R}^{n}} \Delta^{2} u u_{i} d x= \\
& =\int_{\mathbb{R}^{n}}|u|^{2_{*}-2} u u_{i} d x \leq \int_{\mathbb{R}^{n}}\left|u_{i}\right|^{2_{*}} d x=\left\|u_{i}\right\|_{2_{*}}^{2_{*}},
\end{aligned}
$$

which implies $\left\|u_{i}\right\|_{2 *}^{2} \geq S^{(n-4) / 4}$ for $i=1,2$. Hence, again by (15), one obtains

$$
\begin{aligned}
\frac{\|\Delta u\|_{2}^{2}}{\|u\|_{2_{*}}^{2}} & =\left[\int_{\mathbb{R}^{n}}|\Delta u|^{2} d x\right]\left[\int_{\mathbb{R}^{n}}|u|^{2_{*}} d x\right]^{-2 / 2_{*}}=\left[\int_{\mathbb{R}^{n}}|\Delta u|^{2} d x\right]^{4 / n}= \\
& =\left[\int_{\mathbb{R}^{n}}\left|\Delta u_{1}\right|^{2} d x+\int_{\mathbb{R}^{n}}\left|\Delta u_{2}\right|^{2} d x\right]^{4 / n} \geq \\
& \geq\left[S\left\|u_{1}\right\|_{2_{*}}^{2}+S\left\|u_{2}\right\|_{2_{*}}^{2}\right]^{4 / n} \geq 2^{4 / n} S,
\end{aligned}
$$

which concludes the proof.

In a completely similar fashion, one may extend the previous result to any domain in case of Navier boundary conditions. For Dirichlet boundary conditions this seems not possible due to the lack of information about the positivity of the corresponding Green's function.

Lemma 3. Let $\Omega$ be a bounded domain of $\mathbb{R}^{n}$ and assume that $u$ solves (5) and changes sign. Then $\|\Delta u\|_{2}^{2} \geq 2^{4 / n} S\|u\|_{2_{*}}^{2}$.

In the case of the half space, by exploiting nonexistence results for positive solutions, we have a stronger result.

Lemma 4. Let $\Omega=\left\{x_{n}>0\right\}$ be the half space and assume that $u \in$ $D^{2,2}(\bar{\Omega})$ solves the equation

$$
\Delta^{2} u=|u|^{8 /(n-4)} u \text { in }\left\{x_{n}>0\right\}
$$

with boundary data either (2) or (3). Then $\|\Delta u\|_{2}^{2} \geq 2^{4 / n} S\|u\|_{2_{*}}^{2}$.

Proof. Notice first that by [27, Theorem 3.3], equation (17)-(2) does not admit positive solutions. A similar nonexistence result holds for boundary conditions (3), see [30, Corollary 1] which may be easily extended to unbounded domains.

Therefore, any nontrivial solution of (17) (with boundary conditions (2) or (3)) necessarily changes sign. We obtain the result if we repeat the argument of the proof of Lemma 2. With Navier boundary conditions this is straightforward, while with Dirichlet boundary data one may invoke Boggio's principle [5] in the half space. 
As pointed out in the introduction, the $H^{2} \cap H_{0}^{1}$-framework is more involved than the $H_{0}^{2}$-case. Therefore, from now on we restrict our attention to the first situation.

Consider the functional $f: \mathscr{V}(\Omega) \rightarrow \mathbb{R}$

$$
f(u)=\int_{\Omega}|\Delta u|^{2} d x
$$

constrained on the manifold

$$
\mathscr{V}(\Omega)=\left\{u \in H^{2} \cap H_{0}^{1}(\Omega): \int_{\Omega}|u|^{2 *} d x=1\right\} .
$$

We say that $\left(u_{h}\right) \subset \mathscr{V}(\Omega)$ is a Palais-Smale sequence for $f$ at level $c$ if

$$
\lim _{h} f\left(u_{h}\right)=c, \quad \lim _{h}\left\|f^{\prime}\left(u_{h}\right)\right\|_{\left(T_{u_{h}} \mathscr{V}(\Omega)\right)^{*}} \rightarrow 0
$$

where $T_{u_{h}} \mathscr{V}(\Omega)$ denotes the tangent space to the manifold $\mathscr{V}(\Omega)$ at $u_{h}$.

We can now state a global compactness result for the biharmonic operator, in the spirit of [37]. Even if the proof is on the lines of that of Struwe, some difficulties arise. In the appendix we give an outline of the proof emphasizing the main differences with respect to second order equations.

Lemma 5. Let $\left(u_{h}\right) \subset \mathscr{V}(\Omega)$ be a Palais-Smale sequence for $f$ at level $c \in$ $\mathbb{R}$. Then, for a suitable subsequence, one has the following alternative: either $\left(u_{h}\right)$ is relatively compact in $H^{2} \cap H_{0}^{1}(\Omega)$ or there exist $k$ nonzero functions $\widehat{u}_{1}, \ldots, \widehat{u}_{k} \in D^{2,2}$, solving either (14) or (17) with boundary condition (2) and a solution $\widehat{u}_{0} \in H^{2} \cap H_{0}^{1}(\Omega)$ of (5) such that

$$
u_{h} \rightarrow \widehat{u}_{0}\left[\sum_{j=0}^{k} \int\left|\widehat{u}_{j}\right|^{2_{*}} d x\right]^{-1 / 2_{*}} \quad \text { in } H^{2} \cap H_{0}^{1}(\Omega)
$$

and

$$
\lim _{h} f\left(u_{h}\right)=\left[\sum_{j=0}^{k} \int\left|\Delta \widehat{u}_{j}\right|^{2} d x\right]\left[\sum_{j=0}^{k} \int\left|\widehat{u}_{j}\right|^{2_{*}} d x\right]^{-2 / 2_{*}} .
$$

The domain of integration for $\widehat{u}_{0}$ is just $\Omega$, while for $\widehat{u}_{1}, \ldots, \widehat{u}_{k}$, it is either a half space or the whole $\mathbb{R}^{n}$.

When working in $H_{0}^{2}(\Omega)$ a similar compactness result holds true with Navier boundary conditions replaced with Dirichlet boundary conditions.

We believe that nontrivial solutions of (17) either with Navier (2) or Dirichlet (3) boundary conditions do not exist and that the previous lemma may be strengthened (see Section 3.1 and 3.2). Nevertheless, Lemma 5 is sufficient to prove the following compactness property in the second critical energy range. 
Lemma 6. Let $\left(u_{h}\right) \subset \mathscr{V}(\Omega)$ be a Palais-Smale sequence for $f$ at level $c \in\left(S, 2^{4 / n} S\right)$. Then, up to a subsequence, $\left(u_{h}\right)$ strongly converges in $H^{2} \cap$ $H_{0}^{1}(\Omega)$.

Proof. Assume by contradiction that the sequence $\left(u_{h}\right)$ is not relatively compact in $H^{2} \cap H_{0}^{1}(\Omega)$. Then, by Lemma 5 one finds functions $\widehat{u}_{0} \in H^{2} \cap$ $H_{0}^{1}(\Omega)$ and $\widehat{u}_{1}, \ldots, \widehat{u}_{k} \in D^{2,2}$ satisfying (20) and (21). Assume first that all $\widehat{u}_{1}, \ldots, \widehat{u}_{k}$ are positive solutions of (14). Then, by Lemma 1 each $\widehat{u}_{j}$ is of type (11) and attains the best Sobolev constant, i.e.

$$
\int_{\mathbb{R}^{n}}\left|\widehat{u}_{j}\right|^{2_{*}} d x=\int_{\mathbb{R}^{n}}\left|\Delta \widehat{u}_{j}\right|^{2} d x=S\left[\int_{\mathbb{R}^{n}}\left|\widehat{u}_{j}\right|^{2_{*}} d x\right]^{2 / 2_{*}}, \quad j=1, \ldots k,
$$

which implies $\int_{\mathbb{R}^{n}}\left|\widehat{u}_{j}\right|^{2_{*}} d x=S^{n / 4}$ for $j=1, \ldots, k$. In particular, one obtains

$$
\lim _{h} f\left(u_{h}\right)=\left[\int_{\Omega}\left|\widehat{u}_{0}\right|^{2 *} d x+k S^{n / 4}\right]^{4 / n} .
$$

If $\widehat{u}_{0} \equiv 0$, we get $f\left(u_{h}\right) \rightarrow k^{4 / n} S$, while if $\widehat{u}_{0} \not \equiv 0$ for each $k \geq 1$ one has

$$
\left[\int_{\Omega}\left|\widehat{u}_{0}\right|^{2 *} d x+k S^{n / 4}\right]^{4 / n}>(k+1)^{4 / n} S .
$$

In any case we have a contradiction with $S<\lim _{h} f\left(u_{h}\right)<2^{4 / n} S$.

We now consider the case in which at least one $\widehat{u}_{j}$ is sign changing or a solution in the half space $\left\{x_{n}>0\right\}$. By Lemmas 2 and 4 we then have for these $\widehat{u}_{j}$ :

$$
\int_{\mathbb{R}^{n}}\left|\widehat{u}_{j}\right|^{2_{*}} d x=\int_{\mathbb{R}^{n}}\left|\Delta \widehat{u}_{j}\right|^{2} d x \geq 2^{4 / n} S\left[\int_{\mathbb{R}^{n}}\left|\widehat{u}_{j}\right|^{2_{*}} d x\right]^{2 / 2_{*}}
$$

and hence $\int_{\mathbb{R}^{n}}\left|\widehat{u}_{j}\right|^{2 *} d x \geq 2 S^{n / 4}$, while for the remaining $\widehat{u}_{j}$, (22) holds true. In any case, we have $\lim _{h} f\left(u_{h}\right) \geq\left(2 S^{n / 4}\right)^{4 / n}=2^{4 / n} S$, again a contradiction.

\section{Proof of Theorems 1 and 2}

The proof of Theorem 1 is by far more involved than the proof of Theorem 2, because the trivial extension of any function $u \in H^{2} \cap H_{0}^{1}(\Omega)$ by 0 does not yield a function in $H^{2}\left(\mathbb{R}^{n}\right)$. In particular, for the space $H_{0}^{2}(\Omega)$ Lemma 7 easily follows by this extension argument. A further difference is that the positivity conclusion of Lemma 3 does not hold in the Dirichlet boundary value case. But in the latter case, one simply has to drop this argument. Therefore we only deal with the proof of Theorem 1 .

For all smooth $\Omega \subseteq \widetilde{\Omega}$ let $\beta: \mathscr{V}(\Omega) \rightarrow \mathbb{R}^{n}$ be the "barycenter" map

$$
\beta(u)=\int_{\Omega} x|u(x)|^{2 *} d x
$$


Since $\widetilde{\Omega}$ is smooth one finds $\widetilde{r}>0$ such that $\widetilde{\Omega}$ is a deformation retract of

$$
\widetilde{\Omega}_{+}=\left\{x \in \mathbb{R}^{n}: d(x, \widetilde{\Omega})<\widetilde{r}\right\} .
$$

First we show that the energy or, equivalently, the optimal Sobolev constants will remain relatively large if we prevent the functions from concentrating "too close" to their domain of definition.

\section{Lemma 7.}

$\gamma=\inf \left\{\inf \left\{f(u): u \in \mathscr{V}(\Omega), \beta(u) \notin \widetilde{\Omega}_{+}\right\}, \Omega\right.$ smooth subset of $\left.\widetilde{\Omega}\right\}>S$.

Proof. Assume by contradiction that for each $\varepsilon>0$ there exists a smooth $\Omega_{\varepsilon} \subseteq \widetilde{\Omega}$ and $u_{\varepsilon} \in H^{2} \cap H_{0}^{1} \cap C^{\infty}\left(\Omega_{\varepsilon}\right)$ such that

$$
\begin{gathered}
\int_{\Omega_{\varepsilon}}\left|u_{\varepsilon}\right|^{2_{*}} d x=1 \\
\int_{\Omega_{\varepsilon}}\left|\Delta u_{\varepsilon}\right|^{2} d x \leq S+\varepsilon \\
\beta\left(u_{\varepsilon}\right) \notin \widetilde{\Omega}_{+} .
\end{gathered}
$$

Let $U_{\varepsilon} \in H^{2}\left(\mathbb{R}^{n}\right)$ be any entire extension of $u_{\varepsilon}$. Since $\Omega_{\varepsilon}$ is smooth, the existence of such an extension is well known. We emphasize that the quantitative properties of $U_{\varepsilon}$ outside $\Omega_{\varepsilon}$ (which are expected to blow up for $\varepsilon \searrow 0$ ) will not be used. Further, let $1_{\Omega_{\varepsilon}}$ be the characteristic function of $\Omega_{\varepsilon}$.

Step I. We claim that $1_{\Omega_{\varepsilon}} U_{\varepsilon} \rightarrow 0$ in $L^{2}\left(\mathbb{R}^{n}\right)$ as $\varepsilon \rightarrow 0$.

By Theorem 5 there exists $C>0$ independent of $\varepsilon$ such that

$$
\forall u \in H^{2} \cap H_{0}^{1}\left(\Omega_{\varepsilon}\right):\|\Delta u\|_{2}^{2} \geq S\|u\|_{2_{*}}^{2}+\frac{1}{C}\|u\|_{1}^{2} .
$$

Putting together (24), (25) and (27), for each $\varepsilon>0$ we have

$$
S+\varepsilon \geq \int_{\Omega_{\varepsilon}}\left|\Delta u_{\varepsilon}\right|^{2} d x \geq S\left\|u_{\varepsilon}\right\|_{2_{*}}^{2}+\frac{1}{C}\left\|u_{\varepsilon}\right\|_{1}^{2}=S+\frac{1}{C}\left\|1_{\Omega_{\varepsilon}} U_{\varepsilon}\right\|_{1}^{2}
$$

so that $\left\|1_{\Omega_{\varepsilon}} U_{\varepsilon}\right\|_{1} \rightarrow 0$ as $\varepsilon \rightarrow 0$. By (24) and classical $L^{p}$-interpolation, we also get $\left\|1_{\Omega_{\varepsilon}} U_{\varepsilon}\right\|_{2} \rightarrow 0$ as $\varepsilon \rightarrow 0$.

Step II. The weak* limit (in the sense of measures) of $1_{\Omega_{\varepsilon}}\left|U_{\varepsilon}\right|^{2_{*}}$ is a Dirac mass.

Integration by part shows that $\left\|\nabla u_{\varepsilon}\right\|_{L^{2}\left(\Omega_{\varepsilon}\right)}^{2} \leq\left\|\Delta u_{\varepsilon}\right\|_{L^{2}\left(\Omega_{\varepsilon}\right)}\left\|u_{\varepsilon}\right\|_{L^{2}\left(\Omega_{\varepsilon}\right)}$. Then by Step I and (25), we infer that

$$
\left\|1_{\Omega_{\varepsilon}} \nabla U_{\varepsilon}\right\|_{L^{2}\left(\mathbb{R}^{n}\right)}=\left\|\nabla u_{\varepsilon}\right\|_{L^{2}\left(\Omega_{\varepsilon}\right)} \rightarrow 0 .
$$


Therefore, taking any $\varphi \in C_{c}^{\infty}\left(\mathbb{R}^{n}\right)$, as $\varepsilon \rightarrow 0$ we find

$$
\int_{\mathbb{R}^{n}} 1_{\Omega_{\varepsilon}}\left|\Delta\left(\varphi U_{\varepsilon}\right)\right|^{2} d x=\int_{\mathbb{R}^{n}} 1_{\Omega_{\varepsilon}}|\varphi|^{2}\left|\Delta U_{\varepsilon}\right|^{2} d x+o(1) .
$$

By the $L^{1}\left(\mathbb{R}^{n}\right)$ boundedness of the sequences $\left(1_{\Omega_{\varepsilon}}\left|U_{\varepsilon}\right|^{2 *}\right)$ and $\left(1_{\Omega_{\varepsilon}}\left|\Delta U_{\varepsilon}\right|^{2}\right)$ there exist two bounded non negative measures $\nu, \mu$ on $\mathbb{R}^{n}$ such that

$$
1_{\Omega_{\varepsilon}}\left|U_{\varepsilon}\right|^{2_{*}} \rightarrow^{*} \nu, \quad 1_{\Omega_{\varepsilon}}\left|\Delta U_{\varepsilon}\right|^{2} \rightarrow^{*} \mu
$$

in the sense of measures. By the Sobolev inequality in $\Omega_{\varepsilon}$ we have

$$
\forall \varepsilon>0: S\left(\int_{\mathbb{R}^{n}} 1_{\Omega_{\varepsilon}}\left|U_{\varepsilon}\right|^{2 *}|\varphi|^{2 *} d x\right)^{2 / 2_{*}} \leq \int_{\mathbb{R}^{n}} 1_{\Omega_{\varepsilon}}\left|\Delta\left(\varphi U_{\varepsilon}\right)\right|^{2} d x
$$

and therefore, by (28) and (29), letting $\varepsilon \rightarrow 0$ yields

$$
S\left(\int_{\mathbb{R}^{n}}|\varphi|^{2_{*}} d \nu\right)^{2 / 2_{*}} \leq \int_{\mathbb{R}^{n}}|\varphi|^{2} d \mu .
$$

By (24) and (25) we also know that

$$
S\left(\int_{\mathbb{R}^{n}} d \nu\right)^{2 / 2_{*}}=\int_{\mathbb{R}^{n}} d \mu .
$$

Hence by [24, Lemma I.2] there exist $\bar{x} \in \overline{\widetilde{\Omega}}$ and $\sigma>0$ such that $\nu=\sigma \delta_{\bar{x}}$. From (24) we see that $\sigma=1$.

The contradiction follows by Step II, since $\beta\left(u_{\varepsilon}\right) \rightarrow \bar{x}$, against (26). This completes the proof.

According to the previous lemma, we may choose $\mu$ such that $S<\mu<$ $\min \left\{2^{4 / n} S, \gamma\right\}$. Let $\varphi \in C_{c}^{\infty}\left(B_{1}(0)\right)$ be such that

$$
\int_{B_{1}(0)}|\varphi|^{2_{*}^{*}} d x=1, \quad \int_{B_{1}(0)}|\Delta \varphi|^{2} d x<\mu .
$$

Define for each $\sigma>0$ and $y \in \mathbb{R}^{n}$ the function $\varphi_{\sigma, y}: \mathbb{R}^{n} \rightarrow \mathbb{R}$ by setting

$$
\varphi_{\sigma, y}(x)=\varphi\left(\frac{x-y}{\sigma}\right)
$$

where $\varphi$ is set to zero outside $B_{1}(0)$. It is readily seen that there exists $\bar{\sigma}>0$ such that $B_{\bar{\sigma}}(y) \subset \widetilde{\Omega}$ and hence $\varphi_{\bar{\sigma}, y} \in C_{c}^{\infty}(\widetilde{\Omega})$ for each $y \in H$. For all $\Omega \subsetneq \widetilde{\Omega}$ let $z_{\Omega} \in D^{2,2}\left(\mathbb{R}^{n}\right)$ be such that $z_{\Omega}=1$ in $\widetilde{\Omega} \backslash \Omega$ and

$$
\int_{\mathbb{R}^{n}}\left|\Delta z_{\Omega}\right|^{2} d x=\operatorname{cap}(\widetilde{\Omega} \backslash \Omega),
$$

see what follows Definition 1. Note that one has

$$
\left(1-z_{\Omega}\right) T_{y}(\varphi) \in H^{2} \cap H_{0}^{1}(\Omega) \quad \forall y \in H,
$$


where $T_{y}(\varphi):=\frac{\varphi_{\bar{\sigma}, y}}{\left\|\varphi_{\bar{\sigma}, y}\right\|_{2_{*}}}$. Moreover, for each $\delta>0$ there exists $\varepsilon>0$ with

$$
\sup _{y \in H}\left\|z_{\Omega} T_{y}(\varphi)\right\|_{H^{2} \cap H_{0}^{1}(\Omega)}<\delta
$$

whenever $\operatorname{cap}(\widetilde{\Omega} \backslash \Omega)<\varepsilon$. Then one gets

$$
\left\|\left(1-z_{\Omega}\right) T_{y}(\varphi)\right\|_{L^{2 *}(\Omega)} \neq 0 \quad \forall y \in H,
$$

if $\varepsilon$ is sufficiently small. So, if we define the map $\Phi_{\Omega}: H \rightarrow \mathscr{V}(\Omega)$ by

$$
\Phi_{\Omega}(y)=\frac{\left(1-z_{\Omega}\right) T_{y}(\varphi)}{\left\|\left(1-z_{\Omega}\right) T_{y}(\varphi)\right\|_{2_{*}}} .
$$

Taking into account (30) and (31), we find $\varepsilon>0$ such that

$$
\sup \left\{f\left(\Phi_{\Omega}(y)\right): y \in H\right\}<\mu
$$

provided that $\operatorname{cap}(\widetilde{\Omega} \backslash \Omega)<\varepsilon$. From now on $\varepsilon$ is fixed subject to the previous restrictions. Let $\Omega \subset \widetilde{\Omega}$ be such that $\operatorname{cap}(\widetilde{\Omega} \backslash \Omega)<\varepsilon$ and $r \in(0, \widetilde{r})$ be such that $\Omega$ is a deformation retract of

$$
\Omega_{+}=\left\{x \in \mathbb{R}^{n}: d(x, \Omega)<r\right\} .
$$

As in Lemma 7, one obtains

$$
\inf \left\{f(u): u \in \mathscr{V}(\Omega), \beta(u) \notin \Omega_{+}\right\}>S .
$$

Notice that

$$
\inf \left\{f(u): u \in \mathscr{V}(\Omega), \beta(u) \notin \Omega_{+}\right\} \leq \sup \left\{f\left(\Phi_{\Omega}(y)\right): y \in H\right\}
$$

otherwise the map $\mathscr{R}: H \times[0,1] \rightarrow \widetilde{\Omega}$ given by

$$
\mathscr{R}(y, t)=(1-t) y+t \beta\left(\Phi_{\Omega}(y)\right) \quad \forall y \in H, \forall t \in[0,1]
$$

would deform $H$ in $\widetilde{\Omega}$ into a subset of $\Omega_{+}$and then into a subset of $\Omega(\Omega$ is a deformation retract of $\Omega_{+}$) contradicting the assumptions. Here, in order to see $\mathscr{R}(y, t) \in \widetilde{\Omega}$, we used the fact that $\operatorname{supp} \Phi_{\Omega}(y) \subset B_{\bar{\sigma}}(y)$ and that, since $\varphi \geq 0, \beta\left(\Phi_{\Omega}(y)\right) \in B_{\bar{\sigma}}(y) \subset \widetilde{\Omega}$.

Therefore, by combining (32), (33) and (34) one gets

$$
\begin{aligned}
& S<\inf \left\{f(u): u \in \mathscr{V}(\Omega), \beta(u) \notin \Omega_{+}\right\} \leq \sup \left\{f\left(\Phi_{\Omega}(y)\right): y \in H\right\} \\
& <\mu<\gamma \leq \inf \left\{f(u): u \in \mathscr{V}(\Omega), \beta(u) \notin \widetilde{\Omega}_{+}\right\} .
\end{aligned}
$$

In what follows we need to find two appropriate levels, such that the corresponding sublevel sets

$$
f^{c}=\{u \in \mathscr{V}(\Omega): f(u) \leq c\}
$$


cannot be deformed into each other. For this purpose let $c_{1}, c_{2}>S$ be such that

$$
\begin{gathered}
c_{1}<\inf \left\{f(u): u \in \mathscr{V}(\Omega), \beta(u) \notin \Omega_{+}\right\}, \\
c_{2}=\sup \left\{f\left(\Phi_{\Omega}(y)\right): y \in H\right\} .
\end{gathered}
$$

Assume by contradiction that there exists a deformation $\vartheta$ of $f^{c_{2}}$ into $f^{c_{1}}$, i.e.

$$
\vartheta: f^{c_{2}} \times[0,1] \rightarrow f^{c_{2}}, \quad \vartheta(., 0)=I d_{f^{c_{2}}} \text { and } \vartheta\left(f^{c_{2}}, 1\right) \subseteq f^{c_{1}}
$$

We define $\mathscr{H}: H \times[0,1] \rightarrow \widetilde{\Omega}$ by setting for each $x \in H$

$$
\mathscr{H}(x, t)= \begin{cases}(1-3 t) x+3 t \beta\left(\Phi_{\Omega}(x)\right) & \text { if } t \in[0,1 / 3] \\ \widetilde{\varrho}\left(\beta\left(\vartheta\left(\Phi_{\Omega}(x), 3 t-1\right)\right)\right) & \text { if } t \in[1 / 3,2 / 3] \\ \widetilde{\varrho}\left(\varrho\left(\beta\left(\vartheta\left(\Phi_{\Omega}(x), 1\right)\right), 3 t-2\right)\right) & \text { if } t \in[2 / 3,1]\end{cases}
$$

where $\widetilde{\varrho}: \widetilde{\Omega}_{+} \rightarrow \widetilde{\Omega}$ is a retraction and $\varrho: \Omega_{+} \times[0,1] \rightarrow \Omega_{+}$is a continuous map with $\varrho(x, 0)=x$ and $\varrho(x, 1) \in \Omega$ for all $x \in \Omega_{+}$. In order to see that $\mathscr{H}(x, t) \in \widetilde{\Omega}$, one should observe that $c_{2}<\gamma$ and $\vartheta\left(\Phi_{\Omega}(x), 3 t-1\right) \in f^{c_{2}}$, hence $\beta\left(\vartheta\left(\Phi_{\Omega}(x), 3 t-1\right)\right) \in \widetilde{\Omega}_{+}$.

As $\vartheta\left(\Phi_{\Omega}(x), 1\right) \in f^{c_{1}}$ for each $x \in H$ and

$$
c_{1}<\inf \left\{f(u): u \in \mathscr{V}(\Omega), \beta(u) \notin \Omega_{+}\right\},
$$

then for each $x \in H$

$$
\beta\left(\vartheta\left(\Phi_{\Omega}(x), 1\right)\right) \in \Omega_{+}
$$

and $\mathscr{H}$ is a deformation of $H$ in $\widetilde{\Omega}$ into a subset of $\Omega$, in contradiction with our assumptions. Then the sublevel set

$$
f^{c_{2}}=\left\{u \in \mathscr{V}(\Omega): f(u) \leq c_{2}\right\}
$$

cannot be deformed into

$$
f^{c_{1}}=\left\{u \in \mathscr{V}(\Omega): f(u) \leq c_{1}\right\} .
$$

Hence, by combining Lemma 6 with the standard deformation lemma (see [31, Theorem 4.6] and also [10, Lemma 27.2], [38, Theorem 3.11]) one obtains a constrained critical point $u_{\Omega}$ such that

$$
S<f\left(u_{\Omega}\right) \leq \sup \left\{f\left(\Phi_{\Omega}(y)\right): y \in H\right\}<\mu<2^{4 / n} S .
$$

Finally, $u_{\Omega}$ does not change sign by Lemma 3 . 


\section{Proof of Theorems 3, 4 and 5}

Proof of Theorem 3. We assume that $\Omega=B=B_{1}(0)$ is the unit ball and that (8) with $\lambda>0$ has a positive solution $u$. As we have already mentioned above, the result by Troy [41, Theorem 1] shows that $u$ is radially symmetric. As the right hand side in (8) is positive, an iterated application of the maximum principle for $-\Delta$ yields that $-\Delta u$ and $u$ are strictly positive and strictly radially decreasing. We will exploit the following Pohožaev type identity, which may be taken e.g. from [42, Lemma 3.9]:

$$
2 \lambda \int_{B} u^{2} d x=\int_{\partial B}\left(\frac{\partial u}{\partial r}\right)\left(\frac{\partial}{\partial r}(-\Delta u)\right) d S .
$$

As $u$ is radially symmetric we may proceed as follows with the term on the right hand side; here, $e_{n}$ denotes the $n$-dimensional volume of the unit ball $B$ and $c(n)$ denotes a generic positive constant which may vary from line to line:

$$
\begin{gathered}
\int_{\partial B}\left(\frac{\partial u}{\partial r}\right)\left(\frac{\partial}{\partial r}(-\Delta u)\right) d S=-n e_{n} \frac{\partial u}{\partial r}(1) \cdot \frac{\partial}{\partial r}(\Delta u)(1) \\
=\frac{1}{n e_{n}}\left(\int_{\partial B}\left(-\frac{\partial u}{\partial r}\right) d S\right)\left(\int_{\partial B}\left(\frac{\partial}{\partial r}(\Delta u)\right) d S\right) \\
=\frac{1}{n e_{n}}\left(\int_{B}(-\Delta u) d x\right)\left(\int_{B} \Delta^{2} u d x\right) .
\end{gathered}
$$

The first integral needs some further consideration. First of all, by making use of homogeneous Navier boundary conditions, we find:

$$
\int_{B}\left(1-|x|^{2}\right) \Delta^{2} u d x=-\sum_{j=1}^{n} \int_{B}\left(-2 x_{j}\right)(\Delta u)_{x_{j}} d x=2 n \int_{B}(-\Delta u) d x .
$$

Furthermore, as we have already mentioned, $u$ and by (8) also $\Delta^{2} u$ are positive and strictly radially decreasing. This fact can be used to get rid of the degenerate weight factor $1-|x|^{2}$ in the previous term:

$$
\begin{aligned}
\int_{B} \Delta^{2} u d x & \leq \int_{|x| \leq 1 / 2} \Delta^{2} u d x+\int_{1 / 2 \leq|x| \leq 1} \Delta^{2} u d x \\
& \leq \int_{|x| \leq 1 / 2} \Delta^{2} u d x+c(n) \Delta^{2} u\left(\frac{1}{2}\right) \\
& \leq \int_{|x| \leq 1 / 2} \Delta^{2} u d x+c(n) \int_{|x| \leq 1 / 2} \Delta^{2} u d x \\
& \leq c(n) \int_{B}\left(1-|x|^{2}\right) \Delta^{2} u d x
\end{aligned}
$$

Combining this estimate with the previous identity, we obtain from (36) and (37):

$$
\lambda \int_{B} u^{2} d x \geq c(n)\left(\int_{B} \Delta^{2} u d x\right)^{2}=c(n)\left\|\Delta^{2} u\right\|_{1}^{2}
$$


By a duality argument and using elliptic estimates, we find

$$
\|u\|_{2} \leq c(n)\left\|\Delta^{2} u\right\|_{1}, \text { provided } n<8 .
$$

Observe that $L^{2}$-estimates for the biharmonic operator under homogeneous Navier boundary conditions follow immediately from the $L^{2}$-estimates for the Laplacian.

Combining (38) and (39), we see that for any positive radial solution $u$ of $(8)$, we have

$$
\lambda \int_{B} u^{2} d x \geq c(n) \int_{B} u^{2} d x
$$

where the constant $c(n)$ is in particular independent of $u$. As $u>0$, we have necessarily $\lambda \geq c(n)$.

Proof of Theorem 4. Assume by contradiction that $u$ is a nontrivial radial solution of (9). According to the remarks in Section 3.2 on the nonexistence of positive solutions, we may assume that $u$ is sign changing. Further, $u$ cannot have infinitely many oscillations near $\partial B$, because in this case, as $u \in C^{4}(\bar{B})$ we would also have $\Delta u=0$ as well as $\frac{d}{d r} \Delta u=0$ on $\partial B$ and could extend $u$ by 0 to the whole of $\mathbb{R}^{n}$ as a solution of the differential equation. The unique continuation property [33] would give the desired contradiction.

As one may replace $u$ with $-u$ if necessary, we may thus assume that there exists a number $a \in(0,1)$ such that

$$
u(x)=0 \text { for }|x|=a, \quad u(x)>0 \text { for } a<|x|<1 ;
$$

moreover, by a Pohožaev-type identity,

$$
u(x)=\nabla u(x)=\nabla^{2} u(x)=0 \text { for }|x|=1 .
$$

Clearly $\frac{d}{d r} u(a) \geq 0$. We define

$$
f(x):=|u(x)|^{2 *-2} u(x)
$$

and compare $u$ with the solution $v$ of

$$
\left\{\begin{array}{l}
\Delta^{2} v(x)=f(x) \quad \text { for } a<|x|<1 \\
v(x)=\nabla v(x)=0 \text { for }|x| \in\{a, 1\}
\end{array}\right.
$$

Obviously $v>0$ on $a<|x|<1$. We now make use of comparison results, which hold true for radial solutions of biharmonic inequalities in the annulus $\{x: a<|x|<1\}$ and can e.g. be found in [8, Section 2]. First one obtains

$$
\frac{d^{2}}{d r^{2}} v(1)>0
$$

Next, as $u$ and $v$ satisfy the same differential equation, if $\frac{d}{d r} u(a)=0$ it follows that $u \equiv v$ while if $\frac{d}{d r} u(a)>0=\frac{d}{d r} v(a)$ we find $u>v$ in $a<|x|<1$. In both cases (41) gives

$$
\frac{d^{2}}{d r^{2}} u(1)>0
$$


and hence a contradiction with (40).

Proof of Theorem 5. We keep $p \in\left[1, \frac{n}{n-4}\right)$ fixed as in the theorem. Let $B=B_{1}(0)$ denote the unit ball, centered at the origin. By scaling and Talenti's comparison principle [40, Theorem 1] it suffices to prove that there exists $C_{B}>0$ with

$$
\|\Delta u\|_{2}^{2} \geq S\|u\|_{2_{*}}^{2}+\frac{1}{C_{B}}\|u\|_{p}^{2}
$$

for each $u \in H^{2} \cap H_{0}^{1}(B)$, which is radially symmetric and radially decreasing with respect to the origin (and hence positive). Let us set

$$
S_{\lambda, p}=\inf _{\substack{u \in H^{2} \cap H_{0}^{1}(B) \\ u \text { radially decreasing }}} \frac{\|\Delta u\|_{2}^{2}-\lambda\|u\|_{p}^{2}}{\|u\|_{2_{*}}^{2}} .
$$

If by contradiction (42) does not hold, one gets

$$
\forall \lambda>0: S_{\lambda, p}<S
$$

Let us fix some $\lambda \in\left(0, \lambda_{1, p}\right)$, where

$$
\lambda_{1, p}:=\inf _{u \in H^{2} \cap H_{0}^{1}(B) \backslash\{0\}} \frac{\|\Delta u\|_{2}^{2}}{\|u\|_{p}^{2}}
$$

is the first positive eigenvalue of

$$
\begin{cases}\Delta^{2} u=\lambda\|u\|_{p}^{2-p}|u|^{p-2} u & \text { in } B \\ u=\Delta u=0 & \text { on } \partial B .\end{cases}
$$

Arguing along the lines of [6] and [15], one finds a smooth positive radial strictly decreasing solution of

$$
\begin{cases}\Delta^{2} u=u^{(n+4) /(n-4)}+\lambda\|u\|_{p}^{2-p}|u|^{p-2} u & \text { in } B \\ u=\Delta u=0 & \text { on } \partial B .\end{cases}
$$

On the other hand, for any positive radially decreasing solution of (45) one has the following variant of Pohožaev's identity:

$$
\lambda\left(2-\frac{n(p-2)}{2 p}\right)\|u\|_{p}^{2}=\int_{\partial B}\left(\frac{\partial u}{\partial r}\right)\left(\frac{\partial}{\partial r}(-\Delta u)\right) d S .
$$

As in the proof of Theorem 3, we conclude

$$
\lambda\|u\|_{p}^{2} \geq c(n, p)\left\|\Delta^{2} u\right\|_{1}^{2} \geq c(n, p)\|u\|_{p}^{2}
$$

as we have assumed that $p<n /(n-4)$. For $\lambda>0$ sufficiently small, we obtain a contradiction. 


\section{Appendix: Proof of Lemma 5}

We prove the corresponding statement for the "free" functional

$$
E_{\Omega}(u)=\frac{1}{2} \int_{\Omega}|\Delta u|^{2} d x-\frac{1}{2_{*}} \int_{\Omega}|u|^{2 *} d x
$$

which is defined on the whole space $H^{2} \cap H_{0}^{1}(\Omega)$. More precisely, we have

Lemma 8. Let $\left(u_{h}\right) \subset H^{2} \cap H_{0}^{1}(\Omega)$ be a Palais-Smale sequence for $E_{\Omega}$ at level $c \in \mathbb{R}$. Then either $\left(u_{h}\right)$ is relatively compact in $H^{2} \cap H_{0}^{1}(\Omega)$ or there exist $k>0$ nonzero functions $\widehat{u}_{j} \in D^{2,2}\left(\Omega_{0, j}\right), j=1, \ldots, k$, with $\Omega_{0, j}$ either the whole $\mathbb{R}^{n}$ or a half space, solving either (14) or (17) with boundary condition (2) and a solution $\widehat{u}_{0} \in H^{2} \cap H_{0}^{1}(\Omega)$ of (5) such that, as $h \rightarrow \infty$ :

$$
\begin{gathered}
u_{h} \rightarrow \widehat{u}_{0} \text { in } H^{2} \cap H_{0}^{1}(\Omega), \\
\left\|\Delta u_{h}\right\|_{2}^{2} \rightarrow\left\|\Delta \widehat{u}_{0}\right\|_{2}^{2}+\sum_{j=1}^{k}\left\|\Delta \widehat{u}_{j}\right\|_{2}^{2}, \quad E_{\Omega}\left(u_{h}\right) \rightarrow E_{\Omega}\left(\widehat{u}_{0}\right)+\sum_{j=1}^{k} E_{\Omega_{0, j}}\left(\widehat{u}_{j}\right) .
\end{gathered}
$$

In the second order situation, the corresponding result is due Struwe [37]. Related generalisations of the Struwe compactness lemma to higher order problems can be found in $[1,3,11,19]$. However, none of these applies directly to our situation. A particular difficulty here arises from the existence of the boundary $\partial \Omega$ in combination with Navier boundary conditions.

\section{Proof of Lemma 8.}

Step I. Reduction to the case $u_{h} \rightarrow 0$.

By well known arguments [6] we know that there exists $u \in H^{2} \cap H_{0}^{1}(\Omega)$ such that $u_{h} \rightarrow u$ (up to a subsequence) and $E_{\Omega}^{\prime}(u)=0$, namely $u$ solves (5). Moreover, by the Brezis-Lieb lemma we infer that $\left(u_{h}-u\right)$ is again a Palais-Smale sequence for $E$. Therefore we may assume that

$$
u_{h} \rightarrow 0, \quad E\left(u_{h}\right) \rightarrow c \geq \frac{2}{n} S^{n / 4} .
$$

Indeed, if $c<\frac{2}{n} S^{n / 4}$, then we are in the compactness range of $E$ and by arguing as in [6], $u_{h} \rightarrow 0$ up to a subsequence. In this case the statement follows with $k=0$.

Assuming (48) and arguing as for the proof of [37, (3.2), p.187] we deduce

$$
\int_{\Omega}\left|\Delta u_{h}\right|^{2} d x \geq S^{n / 4}+o(1)
$$

Let $L \in \mathbb{N}$ be such that $B_{2}(0)$ is covered by $L$ balls of radius 1 . By continuity of the maps

$$
R \mapsto \sup _{y \in \Omega} \int_{B_{R^{-1}}(y) \cap \Omega}\left|\Delta u_{h}\right|^{2} d x, \quad y \mapsto \int_{B_{R^{-1}}(y) \cap \Omega}\left|\Delta u_{h}\right|^{2} d x
$$


and (49), for $h$ large enough one finds $R_{h}>2 / \operatorname{diam}(\Omega)$ and $x^{h} \in \bar{\Omega}$ such that

$$
\int_{B_{R_{h}^{-1}}\left(x^{h}\right) \cap \Omega}\left|\Delta u_{h}\right|^{2} d x=\sup _{y \in \Omega} \int_{B_{R_{h}^{-1}}(y) \cap \Omega}\left|\Delta u_{h}\right|^{2} d x=\frac{1}{2 L} S^{n / 4} .
$$

When passing to a suitable subsequence, three cases may now occur:

Case I. $R_{h} \nearrow+\infty$ and $\left(R_{h} d\left(x^{h}, \partial \Omega\right)\right)$ is bounded;

Case II. $\left(R_{h} d\left(x^{h}, \partial \Omega\right)\right) \rightarrow+\infty$;

Case III. $\left(R_{h}\right)$ is bounded.

Step II. Preliminaries for Case I.

For every $x \in \mathbb{R}^{n}$ let us denote by $x^{\prime}$ its projection onto $\mathbb{R}^{n-1}$, so that $x=\left(x^{\prime}, x_{n}\right)$. Since $d\left(x^{h}, \partial \Omega\right) \rightarrow 0$, up to a subsequence $x^{h} \rightarrow x_{0} \in \partial \Omega$ and $\varrho_{h}:=R_{h} d\left(x^{h}, \partial \Omega\right) \rightarrow \varrho$. Moreover, for sufficiently large $h$ (say $h \geq \bar{h}$ ) there exists a unique $y^{h} \in \partial \Omega$ such that $d\left(x^{h}, \partial \Omega\right)=\left|y^{h}-x^{h}\right|$.

For all $h \geq \bar{h}$, up to a rotation and a translation, we may assume that $y^{h}=0$ and that the tangent hyperplane $H$ to $\partial \Omega$ at 0 has equation $x_{n}=0$ so that $x^{h}-y^{h} \perp H$. Then, by the smoothness of $\partial \Omega$, there exist an $(n-1)-$ dimensional ball $B_{\sigma}(0)$ of radius $\sigma>0$ (independent of $h$ ) and smooth maps $\psi_{h}: \overline{B_{\sigma}(0)} \rightarrow \mathbb{R}$ such that in local orthonormal coordinate systems over the tangent hyperplanes at $y_{h}$, we have:

$$
\partial \Omega \cap\left(\overline{B_{\sigma}(0)} \times[-\sigma, \sigma]\right)=\left\{\left(x^{\prime}, \psi_{h}\left(x^{\prime}\right)\right): x^{\prime} \in B_{\sigma}(0)\right\} .
$$

Furthermore, there exists $C>0$ (independent of $h$ ) such that

$$
\left|\psi_{h}\left(x^{\prime}\right)\right| \leq C\left|x^{\prime}\right|^{2}, \quad\left|\nabla \psi_{h}\left(x^{\prime}\right)\right| \leq C\left|x^{\prime}\right|, \quad\left|D^{2} \psi_{h}\left(x^{\prime}\right)\right| \leq C \quad \forall x^{\prime} \in B_{\sigma}(0) .
$$

We now rescale the domain $\Omega$ by setting

$$
\Omega_{h}=R_{h}\left(\Omega-x^{h}\right),
$$

so that $x^{h}$ is mapped into the origin 0 while the origin is mapped into $\left(0,-\varrho_{h}\right)$ and $(51)$ becomes

$$
\begin{aligned}
& \partial \Omega_{h} \cap\left(\overline{B_{\sigma R_{h}}(0)} \times\left[-\sigma R_{h}-\varrho_{h}, \sigma R_{h}-\varrho_{h}\right]\right)= \\
& =\left\{\left(x^{\prime}, R_{h} \psi_{h}\left(\frac{x^{\prime}}{R_{h}}\right)-\varrho_{h}\right): x^{\prime} \in B_{\sigma R_{h}}(0)\right\} .
\end{aligned}
$$

Let us set

$$
\begin{gathered}
\omega_{h}=\left\{\left(x^{\prime}, x\right) \in \mathbb{R}^{n}: x^{\prime} \in B_{R_{h} \sigma}(0),\right. \\
\left.R_{h} \psi_{h}\left(\frac{x^{\prime}}{R_{h}}\right)-\varrho_{h}<x_{n}<R_{h} \psi_{h}\left(\frac{x^{\prime}}{R_{h}}\right)-\varrho_{h}+R_{h} \delta\right\}
\end{gathered}
$$


where $\delta>0$ and sufficiently small to have $\omega_{h} \subset \Omega_{h}$. Consider the injective map

$$
\begin{aligned}
\chi_{h}: \overline{B_{R_{h} \sigma}(0)} \times\left[-R_{h} \delta, R_{h} \delta\right] & \rightarrow \mathbb{R}^{n} \\
\left(x^{\prime}, x_{n}\right) & \mapsto\left(x^{\prime}, R_{h} \psi_{h}\left(\frac{x^{\prime}}{R_{h}}\right)+x_{n}-\varrho_{h}\right)
\end{aligned}
$$

and its inverse $\chi_{h}^{-1}$. We observe that $\chi_{h}\left(\overline{B_{R_{h} \sigma}(0)} \times\left[0, R_{h} \delta\right]\right)=\overline{\omega_{h}}$ and that $\chi_{h}$ is bijective on these sets. Thanks to (52), after some computations, one sees that $\left(\chi_{h}\right)$ converges in $C_{l o c}^{2}\left(\left\{x_{n} \geq 0\right\}\right)$ to the translation by the vector $(0,-\varrho)$. Therefore,

$$
\Omega_{0}=\left\{\left(x^{\prime}, x_{n}\right) \in \mathbb{R}^{n}: x_{n}>-\varrho\right\}
$$

is the local uniform limit of $\left(\Omega_{h}\right)$. In particular, for every $\varphi \in C_{c}^{\infty}\left(\Omega_{0}\right)$ we also have that $\varphi \in C_{c}^{\infty}\left(\Omega_{h}\right)$ for sufficiently large $h$. This will be used below. Let us now set

$$
v_{h}(x)=R_{h}^{\frac{4-n}{2}} u_{h}\left(x^{h}+\frac{x}{R_{h}}\right),
$$

so that $v_{h} \in H^{2} \cap H_{0}^{1}\left(\Omega_{h}\right)$. By boundedness of $\left(u_{h}\right)$ we infer that there exists $C>0$ such that $\left\|v_{h}\right\|_{H^{2}\left(\Omega_{h}\right)} \leq C$. Let $1_{\Omega_{h}}$ denote the characteristic function of $\Omega_{h}$, then the sequence $\left(1_{\Omega_{h}} v_{h}\right)$ is bounded in $D^{1,2 n /(n-2)}\left(\mathbb{R}^{n}\right)$ (and in $L^{2_{*}}\left(\mathbb{R}^{n}\right)$ ) so that, up to a subsequence, we have

$$
1_{\Omega_{h}} v_{h} \rightarrow v_{0} \quad \text { in } D^{1,2 n /(n-2)} \cap L^{2_{*}}\left(\mathbb{R}^{n}\right)
$$

where $\operatorname{supp}\left(v_{0}\right) \subseteq \overline{\Omega_{0}}$ and $\left.v_{0}\right|_{x_{n}=-\varrho}=0$. Moreover, as $\left(1_{\Omega_{h}} D^{2} v_{h}\right)$ is bounded in $L^{2}\left(\mathbb{R}^{n}\right)$, by weak continuity of distributional derivatives, we deduce

$$
\int_{\Omega_{h}} D_{i j}^{2} v_{h} \varphi d x \rightarrow \int_{\Omega_{0}} D_{i j}^{2} v_{0} \varphi d x
$$

for all $\varphi \in C_{c}^{\infty}\left(\Omega_{0}\right)$ and $i, j=1, \ldots, n$. In particular, $v_{0}$ has in $\Omega_{0}$ second order weak derivatives.

Step III. The limiting function $v_{0}$ in (54) solves (5) in $\Omega_{0}$.

Fix $\varphi \in C_{c}^{\infty}\left(\Omega_{0}\right)$, then for $h$ large enough we have $\operatorname{supp}(\varphi) \subset \Omega_{h}$. Define $\varphi_{h} \in C_{c}^{\infty}(\Omega)$ by setting

$$
\varphi_{h}(x)=R_{h}^{\frac{n-4}{2}} \varphi\left(R_{h}\left(x-x^{h}\right)\right) .
$$

Therefore, $\left(D^{2} \varphi_{h}\right)$ being bounded in $L^{2}(\Omega)$ and taking into account (53), one obtains

$$
\begin{aligned}
o(1)= & E_{\Omega}^{\prime}\left(u_{h}\right)\left(\varphi_{h}\right)=R_{h}^{\frac{n}{2}} \int_{\Omega} \Delta u_{h} \Delta \varphi\left(R_{h}\left(x-x^{h}\right)\right) d x \\
& -R_{h}^{\frac{n-4}{2}} \int_{\Omega}\left|u_{h}\right|^{2 *-2} u_{h} \varphi\left(R_{h}\left(x-x^{h}\right)\right) d x \\
= & \int_{\mathbb{R}^{n}} \Delta v_{h} \Delta \varphi d x-\int_{\mathbb{R}^{n}}\left|v_{h}\right|^{2_{*}-2} v_{h} \varphi d x \\
= & \int_{\mathbb{R}^{n}} \Delta v_{0} \Delta \varphi d x-\int_{\mathbb{R}^{n}}\left|v_{0}\right|^{2_{*}-2} v_{0} \varphi d x+o(1) .
\end{aligned}
$$


Then $v_{0} \in D^{2,2}\left(\overline{\Omega_{0}}\right)$ solves $(5)$ in distributional sense. The delicate point is to see that $\Delta v_{0}=0$ on $\partial \Omega_{0}$. To this end, let $\varphi \in C_{c}^{2}\left(\mathbb{R}^{n}\right)$ with $\varphi=0$ on $\partial \Omega_{0}$ and define

$$
\varphi_{h}(x)=\varphi\left(\chi_{h}^{-1}(x)-(0, \varrho)\right) .
$$

Notice that for $h$ large enough, also $\varphi_{h} \in C_{c}^{2}\left(\mathbb{R}^{n}\right)$ with $\varphi_{h}=0$ on $\partial \Omega_{h}$. Taking into account that $\left(\chi_{h}^{-1}\right)$ tends to the translation by $(0, \varrho)$, we obtain $\varphi_{h} \rightarrow \varphi$ in $C^{2}\left(\mathbb{R}^{n}\right)$ and

$$
\begin{aligned}
& \int_{\Omega_{0}}\left(\Delta v_{0} \Delta \varphi-\left|v_{0}\right|^{2_{*}-2} v_{0} \varphi\right) d x=\lim _{h} \int_{\Omega_{h}}\left(\Delta v_{h} \Delta \varphi_{h}-\left|v_{h}\right|^{2_{*}-2} v_{h} \varphi_{h}\right) d x \\
& =\lim _{h} \int_{\Omega}\left[\Delta u_{h} \Delta\left(R_{h}^{\frac{n-4}{2}} \varphi_{h}\left(R_{h}\left(x-x^{h}\right)\right)\right)-\right. \\
& \left.\quad-\left|u_{h}\right|^{2_{*}-2} u_{h} R_{h}^{\frac{n-4}{2}} \varphi_{h}\left(R_{h}\left(x-x^{h}\right)\right)\right] d x \\
& =\lim _{h} E_{\Omega}^{\prime}\left(u_{h}\right)\left(R_{h}^{\frac{n-4}{2}} \varphi_{h}\left(R_{h}\left(x-x^{h}\right)\right)\right)=0 .
\end{aligned}
$$

Therefore, by extending any given $\varphi \in H_{0}^{1} \cap H^{2}\left(\Omega_{0}\right)$ oddly with respect to $x_{n}-\varrho$ as a function of $H^{2}\left(\mathbb{R}^{n}\right)$ and then by approximating it by a sequence of $C^{2}$ functions $\left(\varphi_{k}\right)$ with $\varphi_{k}=0$ on $\partial \Omega_{0}$, we get

$$
\forall \varphi \in H_{0}^{1} \cap H^{2}\left(\Omega_{0}\right): \int_{\Omega_{0}} \Delta v_{0} \Delta \varphi d x=\int_{\Omega_{0}}\left|v_{0}\right|^{2_{*}-2} v_{0} \varphi d x
$$

which, as pointed out in $\left[4\right.$, p. 221] implies that $v_{0}$ is also a strong solution of $(5)$ in $\Omega_{0}$.

Step IV. The limiting function $v_{0}$ in (54) is nontrivial.

Let $\varphi \in C_{c}^{\infty}\left(\mathbb{R}^{n}\right)$ such that $\Omega_{0} \cap \operatorname{supp} \varphi \neq \emptyset$; then for $h$ large enough, we may define

$$
\widetilde{v}_{0, h}: \overline{\Omega_{h}} \cap \operatorname{supp}(\varphi) \rightarrow \mathbb{R}, \quad \widetilde{v}_{0, h}(x)=v_{0}\left(\chi_{h}^{-1}(x)-(0, \varrho)\right) .
$$

Since $v_{0} \in C^{2}\left(\overline{\Omega_{0}}\right), v_{0}=\Delta v_{0}=0$ on $\partial \Omega_{0}$ and $\left(\chi_{h}\right)$ converges to the translation by the vector $(0,-\varrho)$, we get as $h \rightarrow+\infty$

$$
\begin{array}{r}
\left\|1_{\Omega_{0}} v_{0}-1_{\Omega_{h}} \widetilde{v}_{0, h}\right\|_{L^{2}(\operatorname{supp}(\varphi))}=o(1), \\
\left\|1_{\Omega_{0}} v_{0}-1_{\Omega_{h}} \widetilde{v}_{0, h}\right\|_{L^{2 *}(\operatorname{supp}(\varphi))}=o(1) \\
\left\|1_{\Omega_{0}} \nabla v_{0}-1_{\Omega_{h}} \nabla \widetilde{v}_{0, h}\right\|_{L^{2}(\operatorname{supp}(\varphi))}=o(1), \\
\left\|1_{\Omega_{0}} \Delta v_{0}-1_{\Omega_{h}} \Delta \widetilde{v}_{0, h}\right\|_{L^{2}(\operatorname{supp}(\varphi))}=o(1) .
\end{array}
$$

To symplify the notations, in what follows we omit the $1_{\Omega_{h}}$ in front of $v_{h}, \nabla v_{h}, \Delta v_{h}$ and $1_{\Omega_{0}}$ in front of $v_{0}, \nabla v_{0}, \Delta v_{0}$. Then by (56) and some com- 
putations, one obtains, as $h \rightarrow+\infty$

$$
\begin{aligned}
\int_{\mathbb{R}^{n}}\left|\Delta\left(\varphi v_{0}-\varphi v_{h}\right)\right|^{2} d x=\int_{\Omega_{h} \cap \operatorname{supp}(\varphi)}\left|\Delta\left(\varphi \widetilde{v}_{0, h}-\varphi v_{h}\right)\right|^{2} d x+o(1) \\
\quad \geq S\left(\int_{\Omega_{h} \cap \operatorname{supp}(\varphi)}\left|\varphi\left(\widetilde{v}_{0, h}-v_{0}\right)\right|^{2 *} d x\right)^{2 / 2_{*}}+o(1) \\
\geq S\left(\int_{\mathbb{R}^{n}}\left|\varphi\left(v_{0}-v_{h}\right)\right|^{2_{*}} d x\right)^{2 / 2_{*}}+o(1)
\end{aligned}
$$

By compact embedding one has $v_{h} \rightarrow v_{0}$ in $L_{l o c}^{2}\left(\mathbb{R}^{n}\right)$ and thanks to an integration by parts one gets

$$
\left\|\nabla\left(\varphi\left(v_{h}-v_{0}\right)\right)\right\|_{2} \leq\left\|\varphi\left(v_{h}-v_{0}\right)\right\|_{2}\left\|\Delta\left(\varphi\left(v_{h}-v_{0}\right)\right)\right\|_{2}
$$

and therefore $\nabla v_{h} \rightarrow \nabla v_{0}$ in $L_{l o c}^{2}\left(\mathbb{R}^{n}\right)$. Hence the previous inequality yields

$$
\int_{\mathbb{R}^{n}} \varphi^{2}\left|\Delta\left(v_{0}-v_{h}\right)\right|^{2} d x \geq S\left(\int_{\mathbb{R}^{n}}|\varphi|^{2 *}\left|v_{0}-v_{h}\right|^{2 *} d x\right)^{2 / 2_{*}}+o(1),
$$

which implies

$$
\int_{\mathbb{R}^{n}} \varphi^{2} d \mu \geq S\left(\int_{\mathbb{R}^{n}}|\varphi|^{2 *} d \nu\right)^{2 / 2^{*}},
$$

where $d \mu$ and $d \nu$ denote respectively the weak* limits of $\left|\Delta\left(v_{0}-v_{h}\right)\right|^{2}$ and $\left|v_{0}-v_{h}\right|^{2 *}$ in the sense of measures.

The rest of this step - to show $v_{0} \not \equiv 0$ - is now very closely along the lines of [1, Lemma 2] and [38, p. 173]. We emphasize that here, the normalization condition (50) is exploited.

Step V. In Case II, a solution of (14) appears.

This follows by arguing as in [1], see also [37]. After rescaling as in (53), $\left(v_{h}\right)$ converges to a solution of (14).

Step VI. Case III cannot occur.

By contradiction, assume that $\left(R_{h}\right)$ is bounded. Then, being $R_{h}>$ $2 / \operatorname{diam}(\Omega)$, we may assume that, up to a subsequence,

$$
x^{h} \rightarrow x_{0} \in \bar{\Omega}, \quad R_{h} \rightarrow R_{0}>0 .
$$

Let us set $\Omega_{0}=R_{0}\left(\Omega-x_{0}\right)$ and

$$
v_{h}=R_{0}^{\frac{4-n}{2}} u_{h}\left(x_{0}+\frac{x}{R_{0}}\right)
$$

As in Case I (with the obvious simplifications), one gets for a subsequence $v_{h} \rightarrow v_{0} \in H^{2} \cap H_{0}^{1}\left(\Omega_{0}\right)$ and that $v_{0} \not \equiv 0$ solves $(5)$ in $\Omega_{0}$. But this is absurd since $u_{h} \rightarrow 0$. 
Step VII. Conclusion.

If $\left(u_{h}\right)$ is a Palais-Smale sequence for $E_{\Omega}$, then by Step I its weak limit $\widehat{u}_{0}$ solves $(5)$. By Steps III, IV and V the "remaining part" $\left(u_{h}-\widehat{u}_{0}\right)$ suitably rescaled gives rise to a nontrivial solution $v_{0}$ of (14) (if Case II occurs) or (17) (if Case I occurs). With help of this solution, we construct from $\left(u_{h}-\widehat{u}_{0}\right)$ a new Palais-Smale sequence $\left(w_{h}\right)$ for $E_{\Omega}$ in $H^{2} \cap H_{0}^{1}(\Omega)$ at a strictly lower energy level. In the case where we find the solution $v_{0}$ in the whole space, one proceeds precisely as in [37], cf. also [1]. In the case that $v_{0}$ is a solution of (17) in a half space $\Omega_{0}$ we again have to use the locally deformed versions $\widetilde{v}_{0, h}$ in $\Omega_{h}$ of $v_{0}$. These have been defined in (55). Let $\varphi \in C_{c}^{\infty}\left(\mathbb{R}^{n}\right)$ be any cut-off function with $0 \leq \varphi \leq 1, \varphi=1$ in $B_{1}(0)$ and $\varphi=0$ outside $B_{2}(0)$. We put

$$
w_{h}(x):=\left(u_{h}-\widehat{u}_{0}\right)(x)-R_{h}^{(n-4) / 2} \widetilde{v}_{0, h}\left(R_{h}\left(x-x^{h}\right)\right) \varphi\left(\sqrt{R_{h}}\left(x-x^{h}\right)\right) .
$$

First we remark that $w_{h}$ is indeed well defined as $\varphi=0$ for $R_{h}\left|x-x^{h}\right| \geq$ $2 \sqrt{R_{h}}$ and as the domain of definition of $\widetilde{v}_{0, h}$ grows at rate $R_{h}$. Further we notice that $\chi_{h}$ and $\chi_{h}^{-1}$ converge uniformly to translations even on $B_{R_{h}}(0)$. For this reason we have

$$
\widetilde{v}_{0, h}(\cdot) \varphi\left(\frac{\cdot}{\sqrt{R_{h}}}\right) \rightarrow v_{0} \quad \text { in } D^{2,2}\left(\overline{\Omega_{0}}\right)
$$

and also in $D^{1,2 n /(n-2)}\left(\overline{\Omega_{0}}\right)$ and in $L^{2 *}\left(\Omega_{0}\right)$. Hence, we have

$$
E_{\Omega}\left(w_{h}\right)=E_{\Omega}\left(u_{h}\right)-E_{\Omega}\left(\widehat{u}_{0}\right)-E_{\Omega_{0}}\left(v_{0}\right)+o(1)
$$

and $E_{\Omega}^{\prime}\left(w_{h}\right) \rightarrow 0$ strongly in $\left(H^{2} \cap H_{0}^{1}(\Omega)\right)^{*}$. Now, the same procedure from Steps II to VI is applied to $\left(w_{h}\right)$ instead of $\left(u_{h}-\widehat{u}_{0}\right)$. As $v_{0} \not \equiv 0$ and hence

$$
E_{\Omega_{0}}\left(v_{0}\right) \geq \frac{2}{n} S^{n / 4}
$$

this procedure stops after finitely many iterations.

Acknowledgement. The authors are grateful to Nicola Garofalo for raising their attention on the paper [22] and to the referees for a very careful reading of the manuscript.

\section{References}

1. C.O. Alves, J.M. do Ò, Positive solutions of a fourth-order semilinear problem involving critical growth, preprint, 2001.

2. A. Bahri, J.M. Coron, On a nonlinear elliptic equation involving the critical Sobolev exponent: the effect of the topology of the domain, Comm. Pure Appl. Math. 41, 1988, 253-294.

3. T. Bartsch, T. Weth, M. Willem, A Sobolev inequality with remainder term and critical equations on domains with nontrivial topology for the polyharmonic operator, preprint, 2002. 
4. F. Bernis, J. García Azorero, I. Peral, Existence and multiplicity of nontrivial solutions in semilinear critical problems of fourth order, Adv. Differential Equations 1, 1996, 219-240.

5. T. Boggio, Sulle funzioni di Green d'ordine $m$, Rend. Circ. Mat. Palermo 20, 1905, 97-135.

6. H. Brezis, L. Nirenberg, Positive solutions of nonlinear elliptic equations involving critical Sobolev exponents, Comm. Pure Appl. Math. 36, 1983, 437477.

7. J.-M. Coron, Topologie et cas limite des injections de Sobolev, C. R. Acad. Sci., Paris, Sr. I 299, 1984, 209-212.

8. R. Dalmasso, Elliptic equations of order $2 m$ in annular domains, Trans. Amer. Math. Soc. 347, 1995, 3575-3585.

9. E.N. Dancer, A note on an equation with critical exponent, Bull. London Math. Soc. 20, 1988, 600-602.

10. K. Deimling, Nonlinear Functional Analysis, Springer, Berlin etc. 1985.

11. F. Ebobisse, M.O. Ahmedou, On a nonlinear fourth order elliptic equation involving the critical Sobolev exponent, preprint, 2001.

12. D.E. Edmunds, D. Fortunato, E. Jannelli, Critical exponents, critical dimensions and the biharmonic operator, Arch. Ration. Mech. Anal. 112, 1990, 269-289.

13. F. Gazzola, Critical growth problems for polyharmonic operators, Proc. Royal Soc. Edinburgh Sect. A 128, 1998, 251-263.

14. F. Gazzola, H.-Ch. Grunau, Critical dimensions and higher order Sobolev inequalities with remainder terms, NoDEA Nonlinear Differential Equations Appl. 8, 2001, 35-44.

15. H.-Ch. Grunau, Positive solutions to semilinear polyharmonic Dirichlet problems involving critical Sobolev exponents, Calc. Var. Partial Differential Equations 3, 1995, 243-252.

16. H.-Ch. Grunau, Polyharmonische Dirichletprobleme: Positivität, kritische Exponenten und kritische Dimensionen, Habilitationsschrift, Universität Bayreuth 1996.

17. H.-Ch. Grunau, On a conjecture of P. Pucci and J. Serrin, Analysis 16, 1996, 399-403.

18. H.-Ch. Grunau, G. Sweers, Positivity for perturbations of polyharmonic operators with Dirichlet boundary conditions in two dimensions, Math. Nachr. 179, 1996, 89-102.

19. E. Hebey, F. Robert, Coercivity and Struwe's compactness for Paneitz type operators with constant coefficients, Calc. Var. Partial Differential Equations 13, 2001, 491-517.

20. E. Jannelli, The role played by space dimension in elliptic critical problems, J. Differential Equations 156, 1999, 407-426.

21. B. Kawohl, G. Sweers, On 'anti'-eigenvalues for elliptic systems and a question of McKenna and Walter, Indiana U. Math. J., to appear.

22. C. Kenig, Restriction theorems, Carleman estimates, uniform Sobolev inequalities and unique continuation, in: Harmonic analysis and partial differential equations, (El Escorial, 1987), 69-90, Lect. Notes Math. 1384, Springer, 1989.

23. C.S. Lin, A classification of solutions of a conformally invariant fourth order equation in $\mathbb{R}^{n}$, Comment. Math. Helv. 73, 1998, 206-231.

24. P.L. Lions, The concentration-compactness principle in the calculus of variations. The limit case. I, Rev. Mat. Iberoamericana 1, 1985, 145-201.

25. S. Luckhaus, Existence and regularity of weak solutions to the Dirichlet problem for semilinear elliptic systems of higher order, J. Reine Angew. Math. 306, 1979, 192-207.

26. E. Mitidieri, On the definition of critical dimension, unpublished manuscript, 1993. 
27. E. Mitidieri, A Rellich type identity and applications, Comm. Partial Differential Equations 18, 1993, 125-151.

28. E. Mitidieri, G. Sweers, Weakly coupled elliptic systems and positivity, Math. Nachr. 173, 1995, 259-286.

29. J.J. Moreau, Décomposition orthogonale d'un espace hilbertien selon deux cônes mutuellement polaires, C. R. Acad. Sci. Paris 255, 1962, 238-240.

30. P. Oswald, On a priori estimates for positive solutions of a semilinear biharmonic equation in a ball, Comment. Math. Univ. Carolinae 26, 1985, 565-577.

31. R. Palais, Critical point theory and the minimax principle, Proc. Symp. Pure Math. 15, 1970, 185-212.

32. D. Passaseo, The effect of the domain shape on the existence of positive solutions of the equation $\Delta u+u^{2^{*}-1}=0$, Topol. Meth. Nonlinear Anal. 3, 1994, 27-54.

33. R.N. Pederson, On the unique continuation theorem for certain second and fourth order elliptic equations, Comm. Pure Appl. Math. 11, 1958, 67-80.

34. S.I. Pohožaev, Eigenfunctions of the equation $\Delta u+\lambda f(u)=0$, Soviet. Math. Dokl. 6, 1965, 1408-1411.

35. P. Pucci, J. Serrin, A general variational identity, Indiana Univ. Math. J. 35, 1986, 681-703.

36. P. Pucci, J. Serrin, Critical exponents and critical dimensions for polyharmonic operators, J. Math. Pures Appl. 69, 1990, 55-83.

37. M. Struwe, A global compactness result for elliptic boundary value problems involving limiting nonlinearities, Math Z. 187, 1984, 511-517.

38. M. Struwe, Variational methods, Springer, Berlin etc. 1990.

39. C.A. Swanson, The best Sobolev constant, Applicable Anal. 47, 1992, 227239.

40. G. Talenti, Elliptic equations and rearrangements, Ann. Scuola Norm. Sup. Pisa Cl. Sci. (IV) 3, 1976, 697-718.

41. W.C. Troy, Symmetry properties in systems of semilinear elliptic equations, J. Differential Equations 42, 1981, 400-413.

42. R.C.A.M. van der Vorst, Variational identities and applications to differential systems, Arch. Ration. Mech. Anal. 116, 1991, 375-398.

43. R.C.A.M. van der Vorst, Best constant for the embedding of the space $H^{2} \cap$ $H_{0}^{1}(\Omega)$ into $L^{2 N /(N-4)}(\Omega)$, Differential Integral Equations 6, 1993, 259-276.

44. R.C.A.M. van der Vorst, Fourth order elliptic equations with critical growth, C. R. Acad. Sci. Paris, Série I 320, 1995, 295-299. 